\title{
Morphology and Rheology of Nanofilled PP / PVA Blends
}

\begin{abstract}
NATALIA REZANOVA*, YURII BUDASH, VIKTORIIA PLAVAN, OLENA ISHCHENKO, VIKTORIIA BULAKH
Kiev National University of Technologies and Design, Department of Applied Ecology, Technologies of Polymers and Chemical Fibers, 2, Nimerovich-Danchenko Str., 01011, Kiev, Ukraine

The influence of $\mathrm{Ag} / \mathrm{SiO}$ as nanofiller and sodium oleate as compatibilizer and their mixtures on the patterns flow and structure formation processes in thermodynamically incompatible polypropylene/plasticized polyvinylalcohol (PP / PVA) systems were studied. It is found that the change in the sequence of mixing the ingredients of the composition allows adjusting the morphology phase type due to the predominant localization of nanoparticle additives in the melt amount of a component or on the border of phase separation. Injection of $\mathrm{Ag} / \mathrm{SiO}$ to melt PP increases dispersion degree and homogeneity of its distribution in the matrix. Simultaneous use of nanoadditive and sodium oleate as compatibilizer improves the connections between the polymers and promotes thinner and more homogeneous dispersion of the particles of the dispersed phase. It is shown that the effective viscosity of melt nanofilled system is determined by components mixing method: viscosity of melt blend increases when the additive is localized predominantly in the PVA and decreases with the prior injection of PP. The nature of the flow for all researched compositions obeys a power law and almostdoes not depend on the method of mixing. The ability of the longitudinal deformation of ternary blends is deteriorating, but remains sufficient for their processing. Combining nanoadditive with compatibilizer promotes increased elasticity and the melt spinnability of modified blends.
\end{abstract}

Keywords: polymer blends, nanoadditives, melt viscosity, morphology, microscopic studies

At present, polymer materials are widely used due to the complex of valuable characteristics - starting from consumer goods to high-tech products. The injection of nanofillers in polymer melts allows giving them new qualities. Using nanoadditives of different chemical nature and geometrical shape provides a variety of effects: reinforcement [1-2], imparting bactericidal properties [34], increase electrical conductivity [5], electromagnetic wave shielding [6], electrostatic charge dissipation, increased barrier performance [7-8], reduced flammability [9] etc.

Overall the morphology and properties of nanofilled polymer blends, along with the concentration of additives and the chemical nature of the functional groups on the surface of its particles, is largely determined by the thermodynamic compatibility of the components and the predominant localization of nanoparticles (NP) in the amount of one component or at the interface. Since the polymers are generally incompatible with each other, they are characterized by a structure that consists of separate phases which properties are determined by the degree of dispersion of the particles of the dispersed phase component and the nanoadditive nature of the distribution. Optimal placement of the nanoparticles determines the valuable qualities of the polymer composition without losing its essential characteristics.

NP migration and localization in polymer blends is experimentally confirmed and depends on thermodynamic and kinetic factors. Filler distribution in the incompatible blends takes place so as to minimize the overall free energy of the system. In other words, the uneven distribution of NP in the composition provides a reduction in free energy, which depends on the surface tension or surface free energy components. Thermodynamically equilibrium localization of solid particles in a multiphase mixture of polymers can be predicted by knowing the interfacial tension between polymers and a solid [10]. Distribution of NP in blends of polymers produced by conventional methods is often different from that attained at thermal equilibrium. However, NP redistribution is also possible by changing the method of mixing components and brownian motion. This distinguishes nanocomposites from composite materials that contain fillers with a particle size greater than $1 \mu \mathrm{m}$.

Final distribution of nanoadditives depends on the mixing conditions (sequence of administration of the ingredients, equipment, temperature and time) and rheological properties of polymers. On the other hand, localization of fillers in blends is determined by the degree of interaction between the components and NP [11-12]. At the same time, the selective localization of NP determines the final morphology and properties of the filled polymer dispersions.

Objective of this study was to research the impact of nanoadditive injection method on the extrudates microstructure and rheological properties of the meltblends of PP/PVA.

\section{Experimental part}

Materials

The following materials were used in the study: polypropylene (PP) and polyvinyl alcohol (PVA), plasticized with glycerol, added in an amount of $10 \mathrm{wt} \%$, and a blend of PP/PVA composition 30/70 by weight \%. For research PP 575P manufactured by «SABIC» and PVA brand «Moviol 5-88» manufactured by «Kuraray Co. Ltd» with a degree of hydrolysis $(86.7 \div 88.7) \%$ were used. Silver/silica $(\mathrm{Ag} /$ $\mathrm{SiO}_{2}$ ) bifunctional compound, which was added in an amount of $5 \mathrm{wt}$.\% by weight of the PP in the blend, was used as a nanoadditive. $\mathrm{Ag} / \mathrm{SiO}_{2}$ was chosen as the nanoadditive because it has a number of advantages compared with the initial antibacterial components: prolonged antimicrobial effect in combination with high sorption and antitoxic properties of silica [4]. Additive Ag/ $\mathrm{SiO}$, was synthesized using Biosil pyrogenic silica by restoring glucose ions $\mathrm{Ag}+$, deposited from aqueousalcoholic solution of silver nitrate on the surface of silica particles. Nanoadditive had the following characteristics: content of silver oxide on the surface $-16.3 \mathrm{~g} / \mathrm{m}^{2}$; size

\footnotetext{
* email: mfibers@ukr.net; Phone: +380959334934
} 
silver NP - $17 \mathrm{~nm}$; surface area - $296 \mathrm{~m}^{2} / \mathrm{g}$. As compatibilizer sodium oleate was used with a content of the main substance of $98.0 \%$ and a molecular weight of 304. The concentration of sodium oleate was $3.0 \mathrm{wt}$. \% by PP weight.

\section{Methods for obtaining blends}

Mixing of polymers and the injection of additives was carried outusing a combined worm-disk extruder LGP 25. Nanofilled blends were prepared in three ways: preliminary dispersion of the nanoadditive in melt PP (1) or melt PVA (2), followed by mixing the granules with dispersed phase of polymer or matrix; (3) - all components were mixed simultaneously. 4-component blend was obtained by injection of nanoadditive, compatibilizer and PP in the melt. The modified granules were mixed with PVA.

\section{Laboratory test}

The viscosity $(\eta)$ of melt blend was studied by capillary viscometer using micro viscometer MV-2 in the range of shear stress $(\tau)(0.1 \div 5.69) \cdot 10^{4} \mathrm{~Pa}$ at a temperature of $190^{\circ} \mathrm{C}$.

Flow regime $(n)$ was determined from the slope of the tangent at a given point of the flow curve to the abscissa.

Elastic properties were evaluated for swelling equilibrium $(B)$ of extrudates annealed as described in the work [13].

The aptitude of the meltfor the longitudinal deformation (spinnability) was determined by the size of the die maximum drawing $\left(D R_{\max }\right)$. It was calculated as the ratio of the highest rate of acceptance of the melt jet to the speed of its outflow from the die.

Quantitative and qualitative assessment of the size and shape of the dispersed phase particles were performed by image analysis of digital photomicrographs of cross sections of the extrudates in the Imagej program. To do this, the selection of objects produced images that relate to the structure of an element by its transformation into a binary division by a threshold procedure. The area $\left(S_{p}\right)$, perimeter $\left(P_{p}\right)$, the equivalent diameter $\left(D_{e}=\left(4 S_{p} / \pi\right)^{0.5}\right)^{\text {, }}$, and shape factor $\left(S F=4 \pi . S_{p} / P_{p}{ }^{2}\right)$ of the dispersed phase were determined.

The quality of additive dispersion in raw polymers was assessed by mixing index $\left(K_{n}\right)$, which is the ratio of the standard deviation of the concentration of particles in clusters $(S)$ to their average concentration in the sample $\left(P_{m}\right)$ :

$$
K_{n}=S / P_{m} \cdot 100 \%
$$

\section{Results and discussions}

Conducted morphometric analysis of nanoadditive Ag/ $\mathrm{SiO}_{2}$ powder indicates that its particles are large agglomerates with a broad size distribution. The largest share of the particles ( $26 \%$ ) corresponds to the range $D_{e}(3 \div 5) \mu \mathrm{m}$. For the bulk of the particles (55 $\%), D_{e}^{e}$ is in the range $(3 \div 9) \mu \mathrm{m}$. Shares of partícles with $D_{e}>9 \mu \mathrm{m}$ and $D_{e}<3 \mu \mathrm{m}$ are significantly different ${ }^{e}(40$ and $5 \%$ respectively). The existence of the «tail» of the distribution of values in the above average can be explained by the presence of particle agglomerates $\mathrm{Ag} / \mathrm{SiO}$ of various sizes. SF index for the primary part particles $(66 \%)$ is in the range $0.4 \div 0.7$. The share of the particles with a shape close to a circular ( $S F>0.8)$ and the share of particles greatly anisometric $(S F<0.2)$ are approximately the same $(\sim$ $5 \%)$.

Results of rheological studies indicate that the injection of nanoadditive increases the initial effective melt viscosity of PP and PVA by 1.1 and 1.7 times, respectively (table 1).

This is due to the effect of solid filling, and for the PVA also structuring the melt due to the formation of hydrogen bonds between the silane and the silanol groups of the silica and polymer macromolecules. The character of the flow of the modified melt is not changed, the melt remains a typical non-Newtonian fluid (index $n$ is in the range $1.5 \div$ 2.2). In the presence of additives, elasticity is reduced by limiting the mobility of macromolecular chains. Based on the values of $D R_{m a x^{\prime}}$ the longitudinal deformation ability is reducing, which may be due to increased system inhomogeneity.

The effective viscosity of melt PP/PVA drops sharply, compared with $\eta$ initial polymers. This regularity is due to the thermodynamic incompatibility between the PP and PVA macromolecules at the interface. The sequence of mixing the components determines the pattern of change in the macro melt rheology of nanofilled compositions.

By injection of the nanoadditive component to the disperse phase, viscosity of the blend decreases sharply. Pre-addition of NP in melt PVA or simultaneously mixing of the ingredients are leads to increase of its viscosity. These results can be explained by the fact that the melt viscosity of the blends is mainly determined by two competing factors: the change of structure formation process of the dispersed phase component in the presence of solid nanoparticles and interaction between functional groups of polymer additives and macromolecules. The dominant influence of one of the processes is related to the possibility of localization of nanoparticles in a volume of dispersed phase, the matrix or at the interface.

\begin{tabular}{|c|c|c|c|c|}
\hline Sample name & $\eta, \mathrm{Pa} \cdot \mathrm{s}$ & $\mathrm{n}$ & $\mathrm{B}$ & $\mathrm{DR}_{\max }$ \\
\hline PP & 290 & 2.1 & 1.5 & 208 \\
\hline $\mathrm{PP}+\mathrm{Ag} / \mathrm{SiO}_{2}$ & 330 & 2.2 & 1.3 & 133 \\
\hline PVA & 270 & 1.5 & 1.7 & 163 \\
\hline $\mathrm{PVA}+\mathrm{Ag} / \mathrm{SiO}_{2}$ & 450 & 1.7 & 1.4 & 960 \\
\hline PP/PVA & 110 & 2.1 & 2.4 & 87 \\
\hline$\left(\mathrm{PP}+\mathrm{Ag} / \mathrm{SiO}_{2}\right)+\mathrm{PVA}$ & 140 & 1.8 & 3.2 & 81 \\
\hline$\left(\mathrm{PVA}+\mathrm{Ag} / \mathrm{SiO}_{2}\right)+\mathrm{PP}$ & 440 & 2.0 & 2.1 & 71 \\
\hline $\mathrm{PP}+\mathrm{PVA}+\mathrm{Ag} / \mathrm{SiO}_{2}$ & 410 & 1.9 & 2.5 & 76 \\
\hline$\left(\mathrm{PP}+\mathrm{Ag} / \mathrm{SiO}_{2}+\right.$ sodium oleate $)+\mathrm{PVA}$ & 120 & 1.8 & 4.1 & 95 \\
\hline
\end{tabular}

Table 1

RHEOLOGICAL PROPERTIES OF THE INITIAL POLYMERSAND PP/PVA BLENDS WITH NANOADDITIVE $\left(\mathrm{Ag} / \mathrm{SiO}_{2}\right)$ 
Completed microscopic studies showed that the Ag/

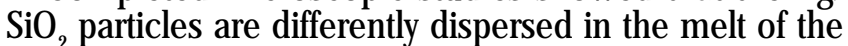
initial components. This is determined by the value of $K_{n}$, which is 27.8 and $11.2 \%$ for meltPP and PVA, respectively. A more uniform distribution of particles in the melt PVA is natural and is due to its high ability to wet the surface of the polar silica particle. When ingredients are mixed by a first method, $\mathrm{Ag} / \mathrm{SiO}_{2}$ is located predominantly in the PP phase and at the interface. This is due to the fact that the injection of the additive with the worst compatibility of NP component is migrating into the interfacial region. NP concentration in it can facilitate the process of change of structure formation in the PP matrix and PVA determines a drop of melt viscosity for the blends obtained by the first method.

The viscosity of compositions can be increased by injection of prior $\mathrm{Ag} / \mathrm{SiO}_{2}$ and PVA into the melt, simultaneously mixing due predominant localization of NP polar additive in volume of polymer matrix and form a network of hydrogen bonds by structuring melt.

The nature of current nanofilled blends, like the original, obeys a power law and is almost does not depend on the mixing sequence of the components. Meltelasticity ternary blends (by value $B$ ) is defined by the sequence of injection of the ingredients, and indirectly indicates a change of structure formation processes in the flow.

The spinnability of all obtained composition melts was reduced, in comparison with the original, due to the increased heterogeneity of systems. But, it is still sufficient for the processing of nanofilled melts in the existing production equipment in fibers and films The simultaneous injection of nanoadditive PP/compatibilizer $(\mathrm{Ag} / \mathrm{SiO}$, sodium oleate) into the melt PP blends provide the fall of viscosity; the spinnability and flexibility are increased, which is an indirect argument of formation for anisotropic structures of the PP in the flow of the compositions (table 1).

It is known that in heterophase polymer blends sizes of disperse phase formations in range from submicron to tens of microns, their shape may be spherical, ellipsoidal, cylindrical, ribbonlike, etc. Analysis of the micrographs of the transverse and longitudinal sections of extrudates shows that the change in the sequence of mixing the ingredients allows to adjust the morphology of the composition of PP/PVA (fig. 1).

In the initial blend PP is crudely dispersed in PVA matrix, there is a substantial amount of film structures, which is conditioned by thermodynamic incompatibility of the components (fig. 1a). With prior injection of $\mathrm{Ag} / \mathrm{SiO}$ in melt $\mathrm{PP}$, dispersed structure type is formed with a uniform distribution of PP particles in PVÀ matrix (fig. 1b). Increase of the dispersion degree is conditioned by the fact that non-polar melt PP poorly wets the hydrophilic additive, and $\mathrm{Ag} / \mathrm{SiO}_{2}$ particles are pushed at the interface reducing the amount of interfacial tension. The ability of NP migration is shown in PE/PLA blends [12]. It is confirmed by electron microscopy that $35 \%$ of the hydrophilic silica NP shifted from PP phase to melt PLA. The majority of the filler particles (65\%) remained in the amount of PE in the form of aggregates. With the injection of nanoadditive to the melt of the polymer matrix, the type of structure is reversed. Formation of hydrogen bonds promotes mutually continuous (interlaced) structures, when it is not possible to determine which polymer is the dispersed phase, and which is the matrix (fig. 1c).

With simultaneous mixing of all components nanoadditive is predominantly localized in the thermodynamically favorable phase (PVA). How ever, the degree of interaction between the blend components is obviously reduced. Pronounced layered structure in the form of concentrically arranged rings was occurred (fig. 1d). Such a change in the morphology of extrudates was also observed in work [9] with polyolefin elastomer/PP blends. With the injection of the silica NP the structure was changed from the interlaced type to the island in the sea type.

Histograms of the distribution of the dispersed phase in terms of $D_{e}$ are shown in the figures 2,3 . They show that with the initial PP blend (fig. 2) forms large agglomerates with a broad size distribution in PVA matrix. $D \sim 80 \%$ of the particles is in the range $(16 \div 44) \mu \mathrm{m}$. For $59 \%$ of the particles, $S F$ is in the range $(0.1 \div 0.8)$; the proportion of the particles close to the round (SF> 0.8) is $41 \%$.

With prior injection of $\mathrm{Ag} / \mathrm{SiO}{ }_{2} \mathrm{PP}$ in the melt PP, the mean value of $D$ particle is reduced by approximately 3.5 times (from $7.30^{\circ}$ to $8.7 \mu \mathrm{m}$ ). For $86 \%$ of the particles it lies within $D 4 \div 11 \mu \mathrm{m}$. In comparison with the previous method of injection, there is a reduction of the standard deviation of the particle distribution by 3.9 times (from 11.6 to 3.0). This indicates an increase in the uniformity of their distribution on this parameter. Dispersed phase distribution SF also changes qualitatively. Almost for all particles SF > 0.8 , and for the majority $(64 \%)$ of the particles it lies in a range SF $0.9 \div 1.0$. Apparently, sharp increase in the dispersion degree of the particles and their approximation
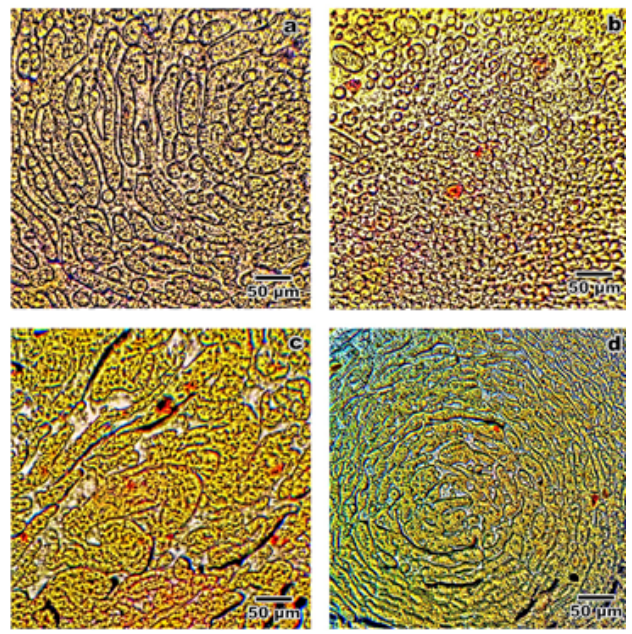

Fig. 1. Micrographs of cross sections of extrudates: (a) the initial $\mathrm{PP} / \mathrm{PVA}$ mixture and nanofilled PP/PVA/Ag/SiO ${ }_{2}$ compositions, obtained by different methods: (b) 1 , (c) 2, (d) 3

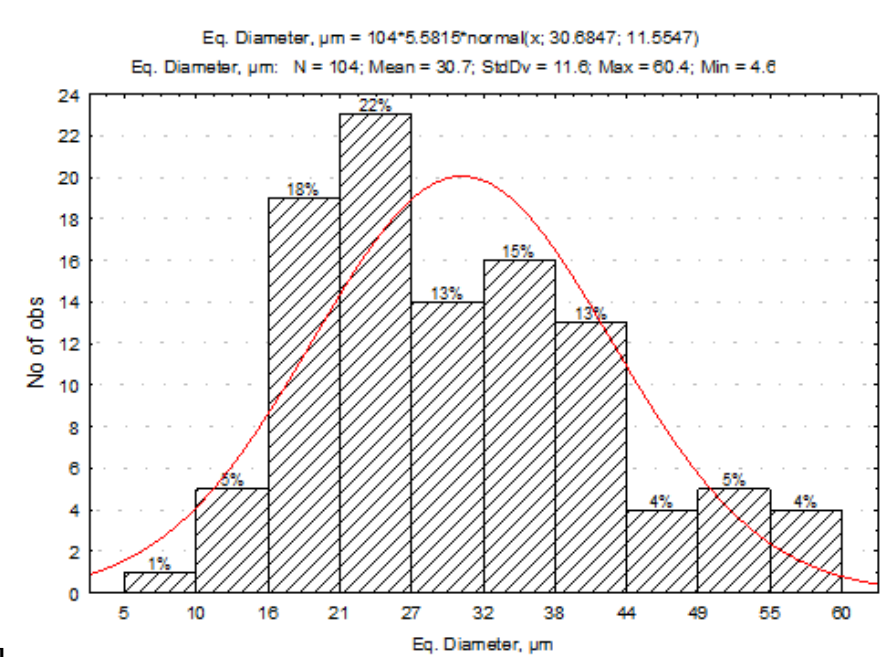

Fig. 2. Histograms of the distribution of the equivalent diameter PP particles in PVA matrix for the initial mixture of PP/PVA. 
Eq. Diameter, $\mu m=402 * 2.2704^{*}$ normal $(x ; 8.7088 ; 2.997)$

Eq. Diameter, $\mu \mathrm{m}: \quad \mathrm{N}=402 ; \mathrm{Mean}=8.7 ; \mathrm{StdDv}=3.0 ; \mathrm{Max}=27.1 ; \mathrm{Min}=4.4$

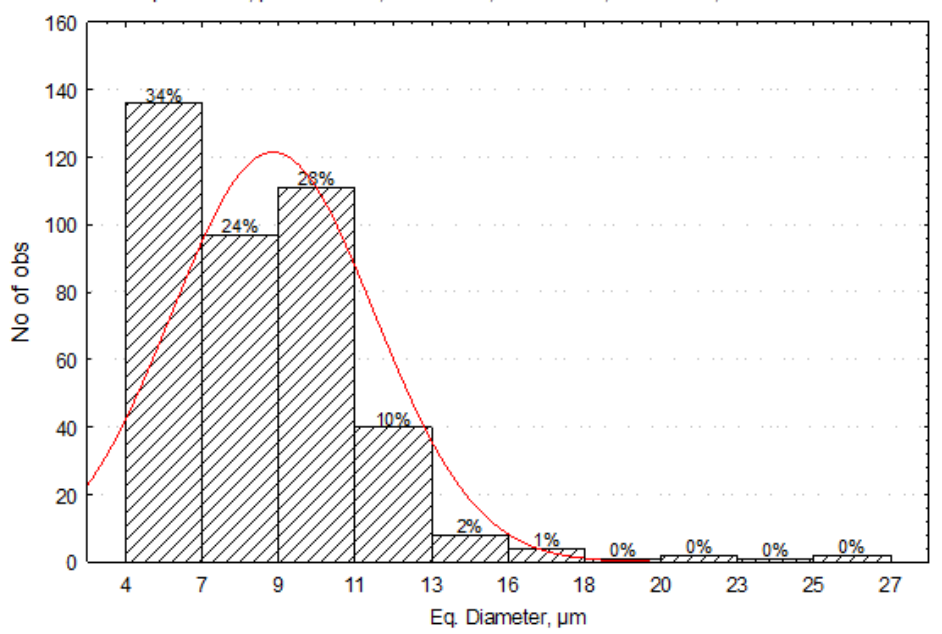

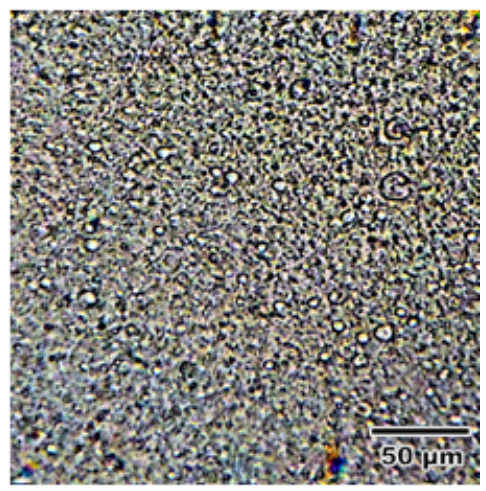

Fig. 4. Micrograph of cross-section of the extrudate fourcomponent composition PP/PVA/( $\left.\mathrm{Ag} / \mathrm{SiO}_{2}\right) /$ sodium oleate

FIg. 3. Histograms of the distribution of the equivalent diameter $\mathrm{PP}$ particles for a mixture of $\mathrm{PP} / \mathrm{PVA} /\left(\mathrm{Ag} / \mathrm{SiO}_{2}\right)$, prepared by method 1

to the right (circular) form in the presence of $\mathrm{Ag} / \mathrm{SiO}$, is the result for improving the compatibility between the PP and plasticized PVA at the interface. This may be a consequence of reducing the surface tension and the cost of a new surface. A similar effect in the presence of silica, carbon black, metal oxides, nanoclays nanoadditives noted in a papers [14-16]. Thus, when 3.0 wt. \% SiO hydrophilic or hydrophobic blend is injection to a polypropylene/ polystyrene (PP/MS), the average particle size of the dispersed phase (PS) is reduced from 3.7 to $0.85 \mu \mathrm{m}$ (for hydrophilic particles $\mathrm{SiO}_{2}$ ) and to $1.25 \mu \mathrm{m}$ (for hydrophobic). This effect is explained by predominant localization of NP in one phase or at the interface. In a preliminary injection of hydrophilic $\mathrm{SiO}_{2}$ in melt PP, NP migrated from PP volume to PS phase. At the same time, NP of hydrophobic $\mathrm{SiO}_{2}$ are displaced in the interfacial region and act as the hard layer which stabilizes and prevents coalescence of the blend of PP/PS [6]. In a blend of PA-6/ABS hydrophilic particles of $\mathrm{SiO}$ are concentrated in PA-6 phase, while the hydrophobic particles are localized at the interface. This reduces the particle size of the copolymer [14].

Our previous studies of the influence of pyrogenic silica on the processes of structure formation in PP/PVA and PP/ copolyamide blends have shown that their use allows obtaining nanofilling PP microfibres with a smaller average diameter and an increased specific surface $[17,18]$.

It is known that the injection of compatibilizer is one of the most effective methods to regulate the morphology of thermodynamically incompatible polymer compositions.

Completed quantitative and qualitative studies of the microstructure of extrudates of 4-component blends show that the simultaneous use of $\mathrm{Ag} / \mathrm{SiO}_{2}$ and sodium oleate contributes to the further growth of the dispersion degree and uniformity of the particle distribution component of the dispersed phase (figs. 4, 5).

The histogram in figure 5 shows that the combined use of $\mathrm{Ag} / \mathrm{SiO}$ and sodium oleate low ers the average particle $D$ approximately by 3.2 times (from 8.7 to $2.7 \mu \mathrm{m}$ ). The standard deviation of the particle distribution is also reduced by 3.9 times (from 11.6 to 3.0). For compositions sodium oleate, $D_{e} 88 \%$ of the particles is in the range $(1 \div 4) \mu \mathrm{m}$, whereas for the compositions without - D 86\% of the particles is in the range of $4 \div 11 \mu \mathrm{m}$. There are no particles larger than $D_{e}>6.0 \mathrm{~mm}$, and the distribution curve is close to symmetric. This is determined by a decrease in asymmetric index distribution by more than 2 times (from 2.0 to 0.9).

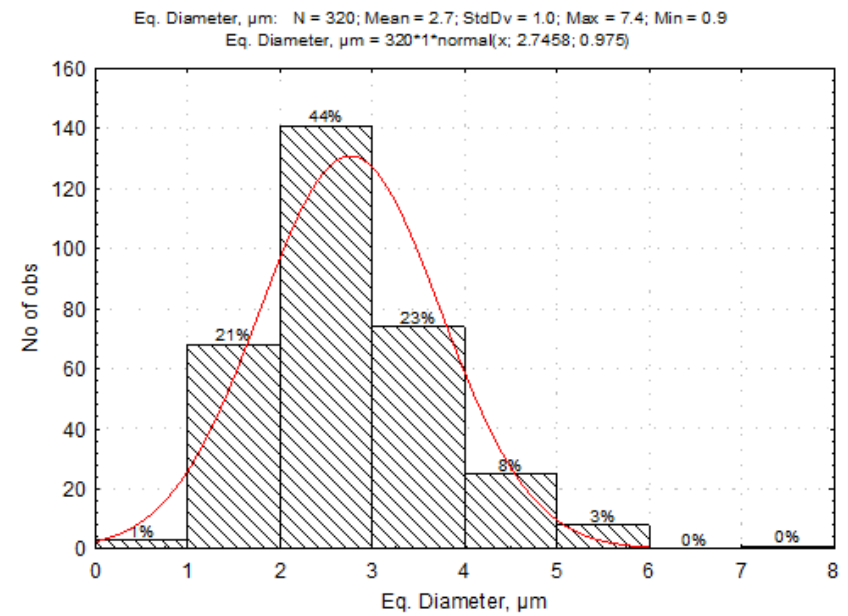

Fig. 5. Histogram of equivalent diameter value distribution of PP particle in PVA matrix for four-component composition PP/PVA/(Ag/ $\mathrm{SiO}_{2}$ )/sodium oleate

The maximum effect of the combined action of nanoadditive and compatibilizer on the structure formation processes can be explained by the decrease of the surface tension at the polymer-polymer and NP-polymer. The affinity between the components due to the formation of specific bonds between the functional groups of the PVA, NP, Ag/ $\mathrm{SiO}_{2}$ and molecules of sodium oleate can be increased.

In order to enhance the influence of the nanoadditive $\left(\mathrm{TiO}_{2}\right)$ on the formation of a matrix-fibril structure in PET/ PP blends the additionally injection of compatibilizer - PP grafted with maleic anhydride was proposed [19].

Simultaneous use of TiO and compatibilizer contributed to reduction of the value of the interfacial tension, increasing degree of deformation of the droplets of the dispersed phase of the component (PET) in the plane and allowed to adjust the diameter and length of the fibrils in the polyester of polypropylene matrix. Reducing the diameter and increasing the length of PET provided improvement of the mechanical properties of composite yarns.

\section{Conclusions}

It is shown that one of the most effective parameters that allow to regulate the micro- and macrorheological properties of melts of mixtures of polypropylene/plasticized polyvinyl alcohol is the selective localization of the $\mathrm{Ag} / \mathrm{SiO}$ nanoadditive in the volume of the melt of the components or at the interface. Changing the sequence of injection of the ingredients ensures the formation of different types of structures - dispersed, interlaced, layered.

It is found that with the prior injection of $\mathrm{Ag} / \mathrm{SiO} \mathrm{NP}_{2}$ in the dispersed phase polymer melt, average particle 
diameter of the PP in the PVA matrix is reduced, compared with the original blends. For three-component compositions $86 \%$ of the particles have an equivalent diameter ranging from 4 to $11 \mu \mathrm{m}$, and the average value of this index decreased by 3.5 times. Reducing the standard deviation of the particle distribution by 3.9 times indicates an increase in the uniformity of their distribution on this indicator.

The combination of nanoadditive and compatibilizer (sodium oleate) promotes further dispersion improvement of the dispersed phase in the matrix. The average value of $D$ particle, the standard deviation and the asymmetry of the distribution of this indicator decreased approximately by 3.2, 3.9 and 2 times, respectively.

It is shown that the rheological properties of nanofilled melts PP/PVA blends are determined by the method of the components mixing. Nanoadditive placement in the matrix promotes the structuring of the melt due to the formation of hydrogen bonds between the functional groups of $\mathrm{SiO}_{2}$ and PVA, which leads to an increase in the effective viscosity. Preliminary injection of nanofiller in PP leads to a sharp decrease in melt viscosity of the blends, due to the formation of dispersed-type structure. Overall, regulation of the localization of the solid nanoadditives in polymer blends (as well as other methods), is a key factor that will generate the desired polymer nanocomposites structure and characteristics.

\section{References}

1.BIANKO S. Carbon Nanotubes - From Research to Applications. Rijeka, Croatia: InTech. - 2011. - 358 p.

2.PAUL D., BUCKNALL C. Polymer blends. New York: John Wiley \& Sons, INC., 2000, p.618.

3.LI W., BURKHART T. Preferential Location of $\mathrm{TiO}_{2}$ Particles in PET/ PP Blend, Proceeding of 19 Congress Francais de Mecanique, August, 2009, Marseille.

4.EGGER S., LEHMAN R.P., HEIGHT M.J., LOESSNER M.J., SCHUPPLER M. Antimicrobial Properties of a Navel Silver-Sliica Nanocomposite Material, Applied and Environmental Microbiology, 75, no. 9, 2009, p. 2973-2976.

5.BUDASH, Y., NOVAK, D., PLAVAN, V. Structural and Morphological Characteristics of Polyethylene Composites with Different Conductive Fillers, Materiale Plastice, 53, no. 4, 2016, p. 693.

6.ELIASL., FENOUILLOT F., MAJ ESTEJ .C., CASSAGNAU PH. Morphology and rheology of immiscible polymer blends filled with silica nanoparticles, Proceeding of 19 Congress Francais de Mecanique, August, 2009, Marseille.
7.XIU H., BAI H. W., HUANG C. M., XU C. L., LI X. Y., FU Q. Selective localization of titanium dioxide nanoparticles at the interface and its effect on the impact toughness of poly(L-lactide)/poly(ether) urethane blends, eXPRESS Polymer Letters, 7, no.3, 2013, p. 261-271.

8.UJ HELYIOVÁ A., STRECKA Z., BOLHOVA E., DULÍKOVA M., BUGAJ P. Polypropylene Fibres Modified by Polyvinyl Alcohol and Nanoadditive. Structure and Properties, Fibres \& Textiles in Eastern Europe, 15, no. 5- 6, 2007, p. 37-40.

9.MORGAN A. B. Flame retarded polymer layered silicate nanocomposites: a review of commercial and open literature systems, Polym. Adv. Technol., no.17, 2006, p. 206-217.

10.FENOUILLOT F., CASSAGNAU P., MAJESTE J.C. Uneven distribution of nanoparticles in inmiscible fluids: Morfology development in polymer blends, Polymer, 50, no.6, p. 1333-1350.

11.LE H.H., LLISCH S., HEIDENREICH D., OSSWALD K., RADUSCH H-J. Phase selective localization of filler in ternary rubber blend, Rubber Chem. Technol., no. 84, 2011, p. 41-53.

12.EBRAHIM JALALI DIL, BASIL D. FAVIS. Localization of micro and nano- silica particles in high interfacial tension poly(lactic acid)/low density polyethylene system, Polymer, no. 77, 2015, p.156-166.

13.UTRACKI L., BAKERDJIANE Z., KAMAL M. A method for the measurement of the true die swell of polymer melts, J. Appl. Polymer Sci, 19, no.2, 1975, p.481-501.

14.CHOW W.S., MOHD ISHAK Z.A. Polyamide blend-based nanocomposites: A review, eXPRESS Polymer Letters, 9, no.3, 2015, p. 232.

15.DOAN V.A., YAMAGUCHI M. Interphase transfer of nanofillers and functional liquid between immiscible polymer pairs, Recent res. Devel. Mat. Sci., 10, 2013, p.59-88.

16.KARGER-KOCSIS J., ZHANG Z. Structure-Properties Relationships in Nanoparticle/Semicrystalline Thermoplastic Composites, In book Mechanical properties of Polymers Based on Nanostructure and Morphology/edited by Michler G.H., Balta-Calleja F.J . Boca Ratio: Taylor \& Francis Group, 2005, p. 456.

17.TSEBRENKO M.V., REZANOVA V.G., TSEBRENKO I.A. Polypropylene microfibers with filler in nano state, Chemistry \& Chemical Technology, 4, no.3, 2010, p.253-260.

18.REZANOVA N.M., REZANOVA V.G., PLAVAN V.P. The influence of nano-additives on the formation of matrix-fibrillar structure in the polymer mixture melts and on the properties of complex threads,Vlakna \& Textil, no. 2, 2017, p. 37-42.

19.LI W., KARGER-KOKSIS J., SCHLARB A.K. Dispersion of $\mathrm{TiO}_{2}$ Particles in PET/PP/TiO and PET/PP/PP-g-MA/TiO ${ }_{2}$ Composites Prepared with Different Blending Procedure, Macromol. Mater. Eng., 294, 2009, p.582-589

$\overline{\text { Manuscript received: } 2.05 .2017}$ 